\title{
How obesity modifies tendons (implications for athletic activities)
}

Michele Abate

Department of Medicine and Science of Aging, University "G. d'Annunzio" Chieti, Pescara, Italy

Corresponding author:

Michele Abate

Department of Medicine and Science of Aging,

University "G. d'Annunzio"

Via dei Vestini 31

66013 Chieti, Pescara, Italy

E-mail:m.abate@unich.it

\section{Summary}

Background: obesity is a well recognized risk factor for dysmetabolic and cardiovascular diseases, but can also be associated to musculo-skeletal disorders.

Methods: a search of English-language articles was performed using the key search terms "obesity" or "body mass index" combined with "tendon", or "tendinopathy", indipendently. Results: several studies show that, in obese subjects, tendons frequently undergo to degeneration, which can progress to a symptomatic stage, with pain and functional impairment. The main histopathologic findings are a relative paucity of small collagen fibrils, expression of an impaired remodeling process, deposition of lipid droplets which can abut to tendolipomatosis, and a disorganized architecture in the tension regions. Both load-bearing and non load-bearing tendons can be affected. This suggests that systemic factors play an important pathogenetic role. Indeed, adipose tissue releases several bioactive peptides and hormones (chemerin, lipocalin, leptin and adiponectin), and cytokines responsible of a systemic state of chronic low grade inflammation. Conclusion: Physical activity is strongly recommended to stop the progression of weight gain or to bring an obese individual into the normal weight range. Therefore, leisure sport activity is useful in obese subjects, but caution is mandatory, because tendons with sub-clinical damage, when submitted to overload, can easily reach the symptomatic threshold.

KEY WORDS: obesity, sport, tendon.

\section{Introduction}

Obesity is a world epidemic, and one of the major public health problems in western countries. It is associated to an increased risk for diabetes, hypertension, and other cardiovascular diseases, as well as for musculo-skeletal disorders ${ }^{1}$. Among the latter, hip and knee osteoarthritis are very common, but several studies suggest that individuals with tendon abnormalities, pain, rupture, or failure to respond to conservative management, have significantly higher adiposity levels than controls without damage ${ }^{2}$. Load-bearing tendons (Achilles, patellar, and plantar fascia) are more frequently involved ${ }^{3-11}$. However, adiposity has been also recognized as a risk factor for alterations in non-load bearing tendons (elbow and rotator cuff) ${ }^{12}$, and a negative impact on the functional outcomes after arthroscopic rotator cuff repair surgery has been reported in obese individuals ${ }^{13,14}$.

Further studies have shown that the probability of tendon abnormalities is higher in males with an increased waist/hip ratio. In a population based study, asymptomatic Achilles tendon pathology was associated with central and peripheral fat distribution in men and women, respectively. It was therefore hypothesized that in men Achilles tendon pathology is linked to metabolic syndrome, whereas in women estrogens, reducing the central accumulation of adipose tissue ${ }^{15}$, may prevent tendon damage. Moreover, it has been shown that tendon disease is also common in individuals who have an increased fat mass and greater waist/hip ratio despite having normal body weight. These subjects, classed as metabolically obese but normal weight, account from 5 to $45 \%$ of the population ${ }^{15,16}$.

Aim of the present paper is to report, according the ethical standard of the Muscles, Ligaments and Tendons Journal17, the more recent findings on the topic, and to suggest convenient strategies to counteract the negative effects of obesity on tendons, mainly in individuals practicing sport activities.

\section{Structural, biochemical and biomechanical features}

The fibrillar tendon composition has been studied in genetically obese Zucker rats. In mice of normal weight, moving free around the cages, the tendons are composed by large and small fibrils, following a bimodal pattern distribution. Large fibrils are essential for the tendon to withstand tension forces, whereas remodeling of the tendon results in the occurrence of 
fibrils with a smaller diameter, indicating the production of new fibrils ${ }^{18,19}$. Conversely, in obese rats, the fibril diameter shows an unimodal distribution, because of the relative prevalence of large on small fibrils, expression of an impaired remodeling process. Because thin fibers confer greater elasticity to tendons, their relative paucity in obese animals could be responsible for increased stiffness and microruptures as a consequence of excessive loads ${ }^{18}$.

Moreover, selective staining procedures show lipid droplets in the extracellular matrix, which could be expression of an early stage of tendolipomatosis, which can progress to severe changes in tendon architecture and function ${ }^{20}$. At ultrastructural analysis by transmission electron microscopy, disorganized and tangled collagen fibrils can be also observed in the tension region of tendons from obese animals 21 . Besides the morphologic abnormalities, biochemical abnormalities have been found. Lower levels of glycosaminoglycans (chondroitin and dermatan sulfate) are present in the deep digital flexor tendon of Zucker rats. Glycosaminoglycans play a vital role in the regulation of tendon extracellular matrix and collagen fibrillogenesis, and therefore their reduced concentration might be responsible for the inadequate deposition and organization of collagen fibrils ${ }^{22}$. On the contrary, a higher hydroxyproline content, found in tendons of obese rats, could be necessary to satisfy the increased mechanical requirements. Indeed, experimental studies in pigs $^{23}$ and chickens ${ }^{24}$ show that tendons with higher hydroxyproline levels are provided of a higher tensile strength associated with lower strain when compared to controls.

Gross anatomy shows that Achilles tendon thickness is significantly higher in obese compared to lean subjects ${ }^{18}$. The larger area partially offsets the greater mass, and therefore the average stress (force per unit area) experienced by the Achilles tendon is similar in both groups. After strenuous exercise (a series of 90-100 repetitions of standing calf raise) tendon thickness is reduced, due to the loss of interstitial water, associated with load-induced alignment of collagen fibers. However, the transverse strain in the tendon, calculated as the natural log of the ratio of post to pre-exercise tendon thickness, in obese subjects is almost half of that of normal weight counterpart. This finding suggest that obesity is associated with structural tendon changes that impair interstitial fluid movement in response to tensile load, and are responsible of a greater transverse stiffness ${ }^{25}$.

\section{Pathogenetic mechanisms}

Prevailing hypotheses of tendon damage in obese subjects are associated with two different mechanisms: the increased yield on the load-bearing tendons and the biochemical alterations attributed to systemic dysmetabolic factors. Indeed, weight-bearing tendons are exposed to higher loads with increasing adiposity, and the higher loads lead to overuse tendinopathy. Alternatively, the systemic hypothesis is based on studies showing that the association with adiposity is equally strong for the non load-bearing and load-bearing tendons ${ }^{26}$.

Adipose tissue is now recognized as a major endocrine and signaling organ, which releases several bioactive peptides and hormones. The adipokinome includes a full range of proteins (such as chemerin, lipocalin 2, serum amyloid A3, leptin and adiponectin) ${ }^{27}$. These proteins influence several activities in various mesenchymal cell phenotypes, which may directly modify tendon structure. In particular, adipokines are able to modulate cytokines, prostanoi$\mathrm{ds}$, and metalloproteinases production ${ }^{28,29}$. The persistently raised serum levels of PGE2, TNF-a, and LTB4, observed in obesity, provide supplementary evidence that a systemic state of chronic, sub-clinic, low-grade inflammation is present in this condition and may act as a prolonged disruptor of tendon homeostasis ${ }^{30-33}$. Moreover, the migration of immune cells, such as macrophages and mast cells, into adipose tissue is associated with a decrease in their circulating levels. As a consequence, the release of profibrotic factors, such as TGF- $\beta$, is reduced, and this may have a detrimental effect on tendon healing, especially if the production of type I and III collagen is also reduced ${ }^{33-35}$. As further consequence, the decreased availability of immune circulating cells should be responsible for less effective immune responses to acute tendon injury ${ }^{36}$.

In subjects with visceral fat, the cluster of metabolic abnormalities is considered the consequence of insulin resistance ${ }^{2}$. Elevated insulin concentrations fail to stimulate increased glucose uptake into muscle, which leads to fasting hyperglycemia, impaired glucose tolerance and eventually type II diabetes mellitus. Advanced Glycation Endproducts (AGEs) formation is markedly accelerated when the availability of blood glucose is increased ${ }^{36,37}$. A key characteristic of reactive AGEs is the formation of covalent crosslinks within collagen fibers, altering their structure and functionality. Indeed, once formed, AGEs can be degraded only when the protein they are linked to is degraded. Other major features of AGEs relate to their interaction with a variety of AGE-binding receptors on the cell surface, which in turn activates several critical molecular pathways and triggers a number of effects. These include pro-oxidant events via generation of reactive oxygen species and further pro-inflammatory events via NFk $\beta$ signaling ${ }^{38}$. This accelerates cross-linking in collagen fibers, leading to sustained up-regulation of pro-inflammatory mediators and a dysfunctional cell phenotype ${ }^{39,40}$. Further negative effects of AGEs include a) modification of shortlived proteins such as Basic Fibroblast Growth Factor, followed by a striking decrease in mytogenic activity, b) intracellular formation of AGEs, leading to destruction of nitric oxide and impaired growth factor signaling, and c) enhanced apoptosis via oxidative stress, increased caspase activity, and extrinsic signaling through pro-apoptotic cytokines. 
Dyslipidemia is another consequence of insulin resistance associated to visceral adiposity ${ }^{15,18}$. It is characterized by elevated total and LDL-cholesterol, low HDL-cholesterol and high tryglicerides levels. However, the deleterious effects of dyslipidemia on tendons are debated. Indeed, studies reporting a lipidic dysmetabolic pattern in subjects with Achilles tendinopathy ${ }^{41}$, and with rotator cuff tears ${ }^{42}$, are challenged by Authors ${ }^{43}$, who did not found any difference among subjects with or without dyslipidemia.

The above reported considerations mainly regard the mechanisms of chronic tendon degeneration. As far as the tendon repair after acute damage is concerned, experiments have been performed on mice, submitted to a high fat diet, and then to flexor digitorum longus (FDL) tendon damage by means of puncturing with a beveled needle ${ }^{44}$. After 28 days, the biomechanical properties of FDL tendons from the obese mice were compromised, with significant reduction of the normalized maximum force, normalized work to maximum force and normalized stiffness, in comparison with normal weight mice submitted to the same experimental procedure. Moreover, histological examination performed at 14 and 28 days showed in obese mice consistently small cellular and fibrous tissue at the injury site, with a lesser degree of collagen remodeling and fiber alignment. These data, applied to humans, could explain the negative impact of obesity on the functional outcomes after surgical repair ${ }^{14,45}$.

\section{Sport activity in obese subjects}

Development of obesity and physical inactivity are closely associated. Obesity rates are higher in sedentary and moderately active persons than in active subjects, as shown by cross-sectional and longitudinal studies ${ }^{1}$. Therefore, physical activity is strongly recommended to slow down, and even stop, the progression of weight gain in obese individuals, or to bring an obese individual into the normal weight range. The weight loss and the active lifestyle reduce considerably the higher risk of diabetes, hypertension, and other cardiovascular diseases, as well of hip and knee osteoarthritis ${ }^{46}$.

Therefore, leisure sport activities are frequently practiced by obese individuals, involved in sedentary work. Cycling, and mainly fast walking and running, are the more popular sports among these subjects. These activities are often performed in 2 or 3 weekly sessions, and not infrequently at high intensity levels to maximize the energy expenditure and to get a significant weight loss. Physicians usually alert their patients about the possible risk of the cardiovascular overload, but less attention is paid to the risk of tendon damage, or even tendon ruptures. This is an important issue, because tendons with sub-clinical damage, when submitted to overload, can reach the symptomatic threshold.

In normal weight subjects, exercise has beneficial ef- fects on tendon morphology and function ${ }^{9}$. It is well known that mechanical loading is essential to maintain tendon homeostasis. When the collagen fibers are stretched, a signal is transmitted via integrins inside the tenocytes, growth factors are released, and the synthesis of proteoglycans and collagen is promoted $^{9,47}$. The effects are balanced by catabolism, due to metalloproteinases and aggrecanases expression. When the mechanical load is repeated and intense, but still in the physiologic window, anabolism prevails on catabolism. New extracellular matrix and collagen fibers are formed, so that, after several months of sustained exercise, tendon cross sectional area increases and the biomechanical properties are improved $^{9}$. However, there is a threshold of loading frequency and magnitude that, once overcome, reverses tendon response from beneficial towards degenerative. An aberration in the proteoglycan metabolism is likely to drive the pathogenesis of tendon damage. Indeed, their exceeding production leads to water retention and swelling, while, at the same time, the increased metalloproteinase expression favours the formation of degradation products. In addition, inflammatory molecules, such as interleukin 1- $\beta$ are released and may be implicated in the disease progression. After the failed healing process, a smoldering fibrogenesis may occur, with matrix turnover and cell activation without normal maturation. Over time, such chronic processes can become symptomatic and lead to altered functioning of the affected tendon. Obviously, tendon components (fibrils, tenocytes, vessels, nerves, etc.) react differently to mechanical loading, depending on the milieu within these components operate. Therefore, it is not surprising that the biochemical milieu, which characterize obese subjects, may have a negative influence on tendon response to loading, favouring degeneration, and/or the evolution to the symptomatic stage of a sub-clinical tendinopathy. These concepts come from studies performed on Achilles tendon in runners ${ }^{9}$. Indeed, during normal running, the tendon is highly solicited and the load can be as high as eight times body weight, so that modest increases in weight are amplified within the tendon ${ }^{48}$. However, they can be applied to all tendons, which are selectively stressed in various athletic activities: the shoulder in swimming and basket, the elbow in tennis and golf, the knee and ankle in all the sports characterized by running and jumping.

\section{Conclusions}

Obesity is characterized by an adverse biochemical milieu, and a chronic low-grade inflammatory state, which may predispose to tendon degeneration. In this condition, physical activity is recommended to reduce excess body weight, prevent body weight regain, and decrease the subsequent risk of developing cardiovascular and orthopedic diseases. In particular, an active lifestyle can significantly reduce tendon degeneration, and even improve symptomatic tendinopathy. 
Therefore, leisure sport activity is useful in overweight or obese subjects. However, it must be kept in mind that an excessive overload can more easily produce pathologic changes, and therefore some caution is necessary. As first advice, the frequency and intensity of the sport performance should be increased gradually, in accordance with the progression of weight loss, avoiding agonistic activity and contrast sports, which are more likely to expose to acute injury. Second, some sports, such as swimming and cycling, which have a minor impact on tendons, should be preferred. However, it is evident that the choice is strictly linked to the pleasure and gratification deriving from the athletic gesture, and therefore highly subjective. In such perspective, every athletic activity may be accepted, providing the strict observance of caution rules.

\section{References}

1. Nantel J, Mathieu ME, Prince F. Physical activity and obesity: biomechanical and physiological key concepts. J Obes. 2011;2011:650230.

2. Gaida JE, Ashe MC, Bass SL, Cook JL. Is adiposity an underrecognized risk factor for tendinopathy? A systematic review. Arthritis Rheum. 2009;61:840-849.

3. Pascual Huerta J, Garcia JM, Matamoros EC, Matamoros JC, Martínez TD. Relationship of body mass index, ankle dorsiflexion, and foot pronation on plantar fascia thickness in healthy, asymptomatic subjects. J Am Podiatr Med Assoc. 2008;98:379-385.

4. Pinzur M, Freeland R, Juknelis $D$. The association between body mass index and foot disorders in diabetic patients. Foot Ankle Int. 2005;26:375-377.

5. Holmes GB, Lin J. Etiologic factors associated with symptomatic achilles tendinopathy. Foot Ankle Int. 2006;27:952-959.

6. Frey C, Zamora J. The effects of obesity on orthopaedic foot and ankle pathology. Foot Ankle Int. 2007;28:996-999.

7. Abate M, Di Carlo L, Salini V, Schiavone C. Metabolic syndrome associated to non-inflammatory Achilles enthesopathy. Clin Rheumatol. 2014.

8. Abate M, Schiavone C, Di Carlo L, Salini V. Achilles tendon and plantar fascia in recently diagnosed type II diabetes: role of body mass index. Clin Rheumatol. 2012;31:1109-1113.

9. Abate M, Oliva F, Schiavone C, Salini V. Achilles tendinopathy in amateur runners: role of adiposity (Tendinopathies and obesity). Muscles Ligaments Tendons J. 2012;2:44-48.

10. Malliaras $\mathrm{P}$, Cook JL, Kent PM. Anthropometric risk factors for patellar tendon injury among volleyball players. $\mathrm{Br} \mathrm{J}$ Sports Med. 2007;41:259-263.

11. Klein EE, Weil L Jr, Weil LS Sr, Fleischer AE. Body mass index and achilles tendonitis: a 10-year retrospective analysis. Foot Ankle Spec. 2013;6:276-282.

12. Rechardt M, Shiri R, Karppinen J, Jula A, Heliövaara M, Viikari-Juntura E. Lifestyle and metabolic factors in relation to shoulder pain and rotator cuff tendinitis: a population-based study. BMC Musculoskelet Disord. 2010;11:165.

13. Wendelboe AM, Hegmann KT, Gren LH, Alder SC, White GL Jr, Lyon JL. Associations between body-mass index and surgery for rotator cuff tendinitis. J Bone Joint Surg Am. 2004;86-A:743-747.

14. Warrender WJ, Brown OL, Abboud JA. Outcomes of arthroscopic rotator cuff repairs in obese patients. J Shoulder Elbow Surg. 2011;20:961-967.

15. Gaida JE, Alfredson H, Kiss ZS, Bass SL, Cook JL. Asymptomatic Achilles tendon pathology is associated with a central fat distribution in men and a peripheral fat distribution in women: a cross sectional study of 298 individuals. BMC Musculoskelet Disord. 2010;11:41.

16. Conus F, Rabasa-Lhoret R, Péronnet F. Characteristics of metabolically obese normal-weight (MONW) subjects. Appl Physiol Nutr Metab. 2007;32:4-12.

17. Padulo J, Oliva F, Frizziero A, Maffulli N. Muscles, Ligaments and Tendons Journal. Basic principles and recommendations in clinical and field science research. MLTJ. 2013;4:250-252.

18. Biancalana A, Veloso LA, Gomes L. Obesity affects collagen fibril diameter and mechanical properties of tendons in Zucker rats. Connect Tissue Res. 2010;51:171-178.

19. Cherdchutham W, Becker CK, Spek ER, Voorhout WF, van Weeren PR. Effects of exercise on the diameter of collagen fibrils in the central core and periphery of the superficial digital flexor tendon in foals. Am J Vet Res. 2001;62:1563-1570.

20. Hills AP, Hennig EM, Byrne NM, Steele JR. The biomechanics of adiposity - structural and functional limitations of obesity and implications for movement. Obes Rev. 2006;7:13-24.

21. Biancalana A, Veloso LA, Taboga SR, Gomes L. Implications of obesity for tendon structure, ultrastructure and biochemistry: a study on Zucker rats. Micron. 2012;43:463-469.

22. Waggett $A D$, Ralphs JR, Kwan AP, Woodnutt D, Benjamin M. Characterization of collagens and proteoglycans at the insertion of the human Achilles tendon. Matrix Biol. 1998;16:457470.

23. Woo SL, Ritter MA, Amiel D, et al. The biomechanical and biochemical properties of swine tendons: long term effects of exercise on the digital extensors. Connect Tissue Res. 1980;7: 177-183.

24. Nakagaki WR, Biancalana A, Benevides GP, Gomes L. Biomechanical and biochemical properties of chicken calcaneal tendon under effect of age and nonforced active exercise. Connect Tissue Res. 2007;48:219-228.

25. Wearing SC, Hooper SL, Grigg NL, Nolan G, Smeathers JE. Overweight and obesity alters the cumulative transverse strain in the Achilles tendon immediately following exercise. J Appl Physiol (1985). 2011;110:1384-1389.

26. Gaida JE, Alfredson L, Kiss ZS, Wilson AM, Alfredson $\mathrm{H}$, Cook JL. Dyslipidemia in Achilles tendinopathy is characteristic of insulin resistance. Med Sci Sports Exerc. 2009;41: 1194-1197.

27. Conde J, Gomez R, Bianco G, et al. Expanding the adipokine network in cartilage: identification and regulation of novel factors in human and murine chondrocytes. Ann Rheum Dis. 2011;70:551-559.

28. Lago R, Gomez R, Otero M, et al. A new player in cartilage homeostasis: adiponectin induces nitric oxide synthase type II and pro-inflammatory cytokines in chondrocytes. Osteoarthritis Cartilage. 2008;16:1101-1109.

29. Berry PA, Jones SW, Cicuttini FM, Wluka AE, Maciewicz RA Temporal relationship between serum adipokines, biomarkers of bone and cartilage turnover, and cartilage volume loss in a population with clinical knee osteoarthritis. Arthritis Rheum. 2011:63:700-707.

30. Cilli F, Khan M, Fu F, Wang JH. Prostaglandin E2 affects proliferation and collagen synthesis by human patellar tendon fibroblasts. Clin J Sport Med. 2004;14:232-236.

31. Cook JL, Purdam CR. Is tendon pathology a continuum? A pathology model to explain the clinical presentation of load-induced tendinopathy. Br J Sports Med. 2009;43:409-416.

32. Maffulli N, Longo UG, Loppini M, Denaro V. Current treatment options for tendinopathy. Expert Opin Pharmacother. 2010;11:2177-2186.

33. Battery L, Maffulli N. Inflammation in overuse tendon injuries. Sports Med Arthrosc. 2011;19:213-217.

34. Scott A, Lian R, Bahr R, Hart DA, Duronio V, Khan KM. Increased mast cell numbers in human patellar tendinosis: cor- 
relation with symptom duration and vascular hyperplasia. $\mathrm{Br} \mathrm{J}$ Sports Med. 2008;42:753-757.

35. Vesentini S, Redaelli A, Gautieri A. Nanomechanics of collagen microfibrils. Muscles, Ligaments and Tendon J. 2013;3 (1):23-34.

36. Del Buono A, Battery L, Denaro V, Maccauro G, Maffulli N. Tendinopathy and inflammation: some truths. Int J Immunopathol Pharmacol. 2011;24:45-50.

37. Gautieri A, Redaelli A, Buehler MJ, Vesentini S. Age -and diabetes- related nonenzymatic crosslinks in collagen fibrils: candidate amino acids involved in Advanced Glycation End-products. Matrix Biol. 2014;34:89-95.

38. Matsuura F, Hirano K, Koseki M, et al. Familial massive tendon xanthomatosis with decreased high-density lipoprotein-mediated cholesterol efflux. Metabolism. 2005;54:1095-1101.

39. Oosterveer DM, Versmissen J, Yazdanpanah M, Defesche JC, Kastelein JJ, Sijbrands EJ. The risk of tendon xanthomas in familial hypercholesterolaemia is influenced by variation in genes of the reverse cholesterol transport pathway and the low-density lipoprotein oxidation pathway. Eur Heart J. 2010;31:1007-1012.

40. Beason DP, Abboud JA, Kuntz AF, Bassora R, Soslowsky LJ. Cumulative effects of hypercholesterolemia on tendon biomechanics in a mouse model. J Orthop Res. 2011;29:380-383.

41. von Bahr S, Movin T, Papadogiannakis N, et al. Mechanism of accumulation of cholesterol and cholestanol in tendons and the role of sterol 27-hydroxylase (CYP27A1). Arterioscler Thromb Vasc Biol. 2002;22:1129-1135.

42. Abboud JA, Kim JS. The effect of hypercholesterolemia on rotator cuff disease. Clin Orthop Relat Res. 2010;468:14931497.

43. Longo UG, Franceschi F, Spiezia F, Forriol F, Maffulli N, Denaro $V$. Triglycerides and total serum cholesterol in rotator cuff tears: do they matter? Br J Sports Med. 2010;44:948-951.

44. David MA, Jones KH, Inzana JA, Zuscik MJ, Awad HA, Mooney RA. Tendon repair is compromised in a high fat dietinduced mouse model of obesity and type 2 diabetes. PLoS One. 2014;9:e91234.

45. Namdari S, Baldwin K, Glaser D, Green A. Does obesity affect early outcome of rotator cuff repair? J Shoulder Elbow Surg. 2010;19:1250-1255.

46. Messier SP, Loeser RF, Miller GD, et al. Exercise and dietary weight loss in overweight and obese older adults with knee osteoarthritis: the Arthritis, Diet, and Activity Promotion Trial. Arthritis Rheum. 2004;50:1501-1510.

47. Birch HL, Thorpe CT, Rumian AP. Specialisation of extracellular matrix for function in tendons and ligaments. MLTJ. 2013;3 (1):12-22.

48. Scott SH, Winter DA. Internal forces of chronic running injury sites. Med Sci Sports Exerc. 1990;22:357-369. 